\title{
Manger ou parler
}

Gérard Couly

$>$ L'ingestion nutritionnelle oro-pharyngée humaine est ancienne, comme celle des mammifères, depuis 150 millions d'années. Cette fonction est assurée et coordonnée par le système nerveux central et périphérique. Or, Manger et parler utilisent les mêmes conduits et voies anatomiques. L'aire praxique ${ }^{1}$ du langage de Broca est contigüe à celle de la commande motrice de la mastication et de la déglutition dans le cortex moteur cérébral. Cette aire praxique de Broca, innovation récente de l'évolution humaine, s'est connectée aux voies motrices anciennes de l'oralité alimentaire qui préexistaient. Le connectome cérébral et le gène Foxp2 ont contribué de manière décisive à ce raccordement pour l'avènement du langage. <

Manger, l'oralité alimentaire, et parler, l'oralité verbale, sont deux activités humaines qui utilisent en commun la même succession de structures anatomiques que sont les lèvres, la bouche, la langue, le voile du palais et le pharynx pour la première, alors que pour la seconde ce sens est inversé afin d'exprimer le langage. Mammifères et oiseaux sont équipés des mêmes dispositifs anatomofonctionnels pour se nourrir et pour émettre leurs cris ou leurs chants. Pour Alain Prochiantz, «le langage est une acquisition récente de l'évolution humaine dont les bases anatomiques et génétiques seraient apparues il y a 600000 ans » [1], ce qui contraste avec l'ancienneté de la nécessité vitale de se nourrir des mammifères depuis 150 millions d'années (MA), ou celle des oiseaux (100 MA). Cette émergence du langage humain a-t-elle bénéficié des fondements anatomiques et praxiques neuro-musculaires orofaciaux anciens de l'oralité alimentaire [2] ? Le connectome cérébral ${ }^{2}$ [3] et le gène Foxp2 (Forkhead-box P2) ${ }^{3}$ [4] ont-ils pu contribuer alors utilement à cette innovation neuro-fonctionnelle?

${ }^{1}$ Relatif à l'action: I'aire praxique du langage est l'aire impliquée dans l'action de parler.

${ }^{2}$ Le connectome est le réseau structurel des circuits intra-cérébraux et de leurs connections, dans lequel circule son trafic de fonctionnement. Le connectome est étudié par imagerie de tenson de diffusion.

${ }^{3}$ Ce gène a été associé à la capacité de parole chez l'homme et au chant des oiseaux.

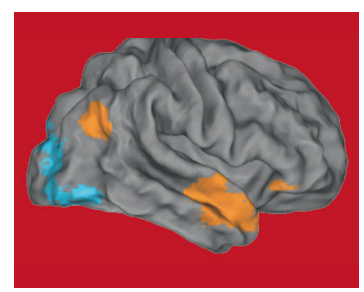

Sorbonne Universités, UPMC Université Paris 06, Institut de la vision, Inserm UMRS 968 , 17 rue Moreau, 75012 Paris, France.

Professeur Honoraire de chirurgie maxillo-faciale de l'enfant Université Paris DescartesSorbonne Paris Cité, Paris, France.

gerard.couly@gmail.com

Le développement de l'oralité alimentaire humaine débute lors de la $12^{\mathrm{e}}$ semaine de gestation, à la fin de la période embryonnaire, par la séquence motrice globale de succion et de déglutition du liquide amniotique, objectivée en échographie fœtale. C'est une activité réflexe et automatique qui implique le développement anatomique des structures orales déjà citées et celui, neuromusculaire, oro-pharyngé dont la commande et la coordination dépendent des noyaux des nerfs crâniens (V trijumeau, VII facial, IX glosso-pharyngien, X pneumogastrique ou vague, $\mathrm{XI}$ spinal bulbaire laryngé, XII grand hypoglosse) et de la substance réticulée du tronc cérébral [5]. À partir de la naissance, cet appareil neuromusculaire permet également la protection des voies respiratoires pendant le nourrissage lacté du nouveau-né par succion-déglutition et l'émission laryngée des cris fréquents de faim.

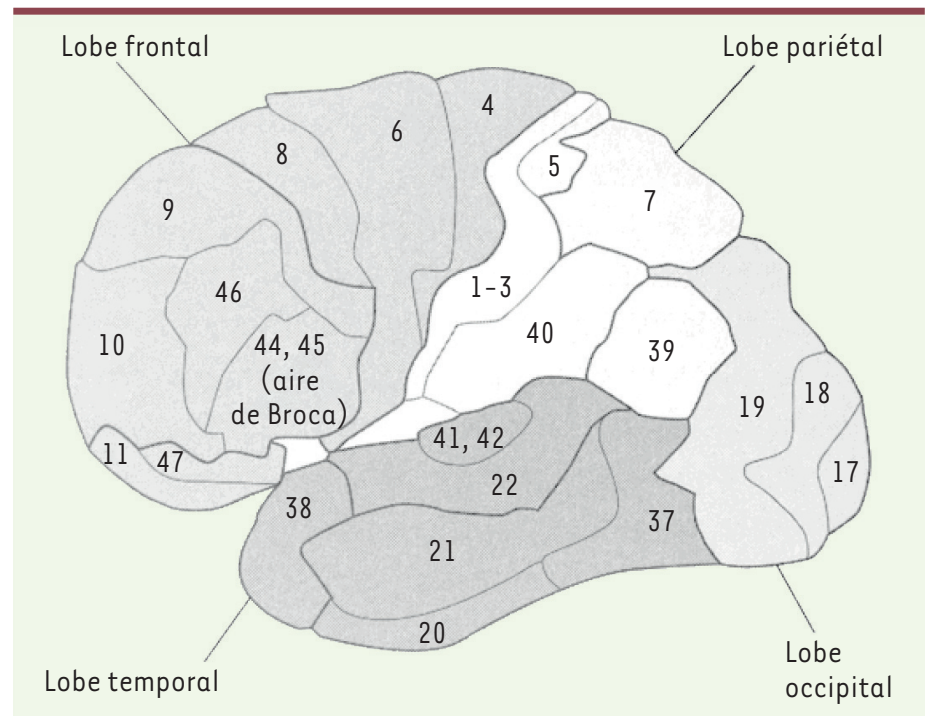

Figure 1. Schéma de la face externe de l'hémisphère cérébral gauche humain objectivant la carte de Brodmann. Les aires 4 et 6 du cortex moteur pré-rolandique (devant la scissure de Rolando, ou sillon central) comporte dans sa partie inférieure la commande motrice de la mastication et de la déglutition. Cette région est contigüe aux aires 44 et 45 praxiques du langage de Broca. 


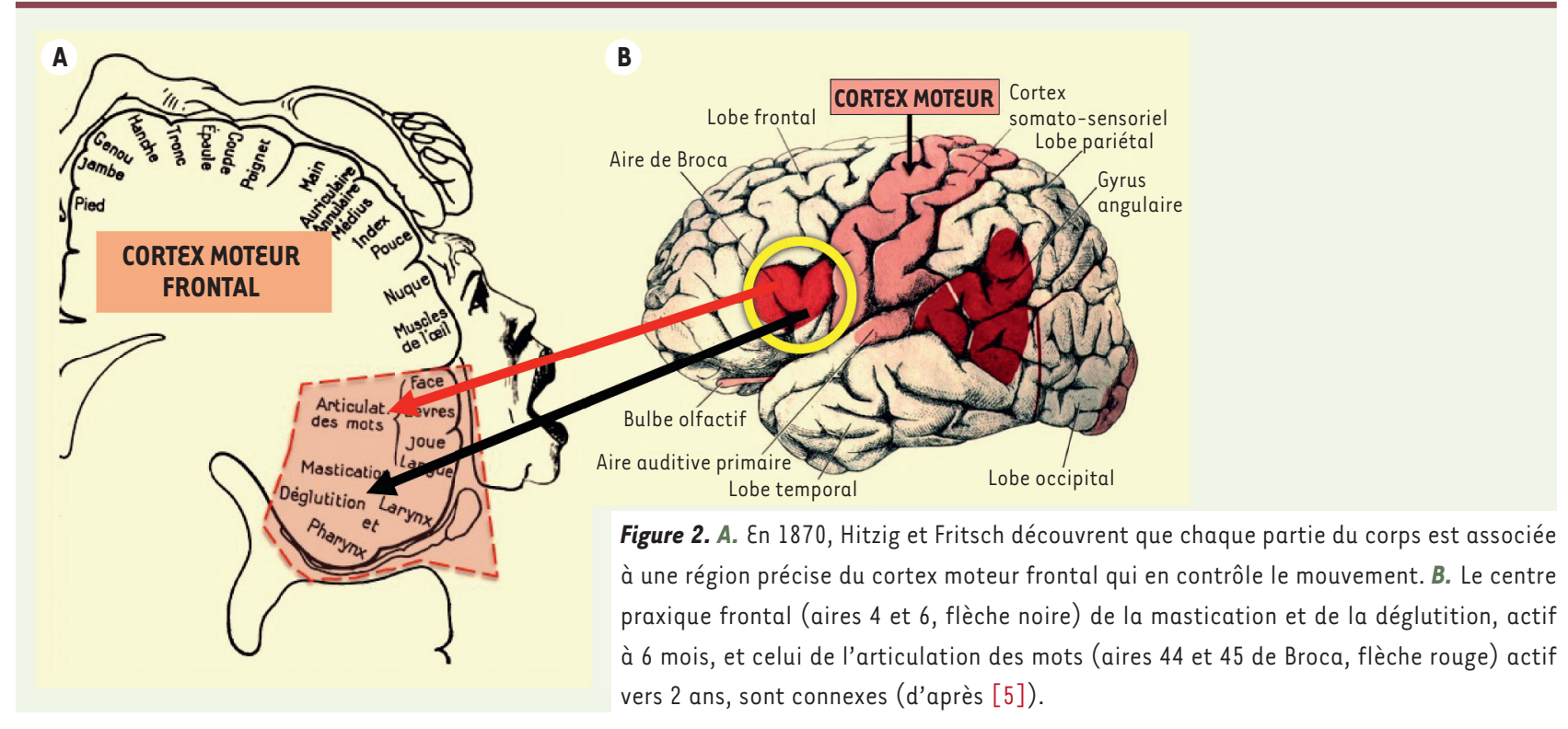

Au cours du $2^{\mathrm{e}}$ semestre post-natal, une nouvelle stratégie alimentaire succède à la succion-déglutition réflexe : c'est la praxie corticale volontaire de mastication. Ces deux stratégies successives de l'oralité nutritive, réflexe puis volontaire, utilisent la même voie d'ingestion orale mais précèdent de deux années l'éclosion du langage [6]. Au cours du $3^{\mathrm{e}}$ semestre post natal, l'enfant commence à reproduire par apprentissage ses premiers mots ou phonèmes grâce à son audition. Les vibrations sonores de l'air expiratoire générées par les replis vocaux endo-laryngés sont émises hors de la bouche (comme les cris) en empruntant de façon inversée la voie anatomique de l'oralité alimentaire (du pharynx aux lèvres) mais en utilisant les mêmes effecteurs neuro-musculaires et organiques. La langue, par exemple, est sollicitée pour réaliser ces deux activités motrices selon qu'elle mastique puis déglutit ou qu'elle participe à l'articulation phonétique. Ainsi l'acte de se nourrir et celui de parler, partagent la même communauté anatomique oro-faciale et la même organisation neuro-musculaire. Chacune des deux oralités l'utilise de façon spécifique et alternative.

L'oralité nutritionnelle a, de par son ancienneté historique puis sa précocité fonctionnelle embryonnaire, rendu possible l'installation puis la pérennité récente de la phonation chez l'enfant au cours de sa deuxième année [6]. François Jacob [7] a nommé cela «le bricolage de l'évolution ». II dit que « cette dernière ne tire pas ses nouveautés du néant. Elle travaille sur ce qui existe déjà, soit qu'elle transforme un système ancien pour lui donner une fonction nouvelle soit qu'elle combine plusieurs système pour en échafauder un autre plus complexe ». L'utilisation par l'oralité alimentaire et l'oralité verbale d'un même appareil neuromusculaire ne fut possible que par la connexité cérébrale de leurs aires praxiques respectives, au niveau du cortex moteur frontal (Figure 1 et 2). C'est ce que nous observons en neuro-anatomie cérébrale humaine [5]. Les aires 4 et 6 praxiques de Brodmann des motricités linguales, masticatrices et déglutitives, dévolues à l'oralité nutritionnelle et localisées dans le pied de la frontale ascendante du cortex moteur pré-rolandique, et les aires 44 et 45 de Brodmann ou aire praxique du langage de Broca, sont bien contigües [5]. Grâce au connectome cérébral ${ }^{4}$ l'aire praxique du langage s'est trouvée «raccordée » au réseau neurologique moteur volontaire préexistant de l'oralité alimentaire. De ces aires motrices partent les voies efférentes descendantes des faisceaux cortico-nucléaires ou géniculés (et également pyramidaux) qui servent de relais synaptiques dans les noyaux des nerfs crâniens du tronc cérébral destinés à la mastication, la déglutition et également à la motricité oro-faciale de la phonation [5]. Or, il est impossible de mastiquer ou de déglutir et de parler simultanément. Les coordinations motrices praxiques spécifiques et en alternance de chacune des deux oralités ont été rendues possible par le connectome cérébral [3].

L'analyse clinique des conséquences motrices, sur l'efficience du langage, de la mutation du gène Foxp2 chez les humains qui en sont atteints $[8,9]$, révèle une dyspraxie du langage dont l'origine est l'aire de Broca, associée à une dyspraxie oro-faciale avec incoordination de l'ingestion alimentaire et fausses routes respiratoires telles que rapportées chez l'enfant [10]. Ces observations objectivent la défaillance commune de la connectivité des fonctions d'ingestion nutritive et langagière de cette mutation.

Ainsi, l'organisation neuro-fonctionnelle puis organique oro-pharyngo-laryngée de la fonction d'ingestion alimentaire a été «utilisée » par cette innovation récente qu'est le langage alors que les origines et le développement de ces deux fonctions sont temporellement

${ }^{4}$ Le connectome [3] est le réseau structurel des circuits intra cérébraux et de leurs connections, dans lequel circule son trafic de fonctionnement. Le connectome est étudié par imagerie de tenson de diffusion. 
très décalés, de plusieurs millions d'années. L'émergence du langage il y a 600000 ans n'a pas eu besoin de structures anatomiques et de l'efficience de circuits neuronaux propres: ceux de l'oralité alimentaire préexistaient. Le connectome et l'aire praxique de Broca ont été, par contre, nécessaires.

Manger, comportement vital et universel des vertébrés, a pu permettre l'émergence de formes variées de communications: cris des mammifères, chant de l'oiseau et langage de l'humain. Manger et parler partagent une communauté universelle plus grande qu'anatomique et neuro-musculaire car nous sommes aptes à manger toutes les cuisines et parler toutes les langues du monde. $\diamond$

\section{SUMMARY}

\section{To eat or to speak}

Human oro-pharyngeal feeding is old as mammals's (150 millions years). This fonction is performed and coordinated by the central and peripheric nervous system. Thus, eating and speaking use the same anatomic ducts and ways. To that purpose, the Broca praxic language area is close to the praxic area of the motor mastication and swallowing control in brain cortex. This area, a new innovation of human evolution, is connected directly with the old motor ways of preexisting oral feeding. The brain connectome and Foxp2 gene have contributed with efficiency to this linking when the language came. $\diamond$
LIENS D’INTÉRÊT

L'auteur déclare n'avoir aucun lien d'intérêt concernant les données publiées dans cet article.

\section{RÉFÉRENCES}

1. Prochiantz A. Singe toi-même. Paris : Éditions Odile Jacob, 2019.

2. Couly G. L'oralité du fœetus. Montpellier : Sauramps, 2015.

3. Seung S. Connectome, how the brain's wiring make us who we are? Boston : Houghton Mifflin Harcourt Trade, 2012.

4. Lai C, Fisher S, Hurst J, et al. A forkhead-domain gene is mutated in a severe speech and language disorder. Nature $2001 ; 413: 519-23$.

5. Delmas J, Delmas A. Voies et centres nerveux, $5^{e}$ ed. Paris : Masson, 1958.

6. Couly G. L'oralité du fœtus, fondement du langage. Réeducation Orthophonique (Paris) $2017 ; 271: 13-27$

7. Jacob F. La souris, la mouche et l'homme. Paris : Éditions Odile Jacob, 1997.

8. Enard W, Przewworski M, Fisher S, et al. Molecular evolution of FOXP2, a gene involved in speech and language. Nature 2002 ; 418: 869-72.

9. Fisher S. Human genetics : the evolving story of FOXP2. Curr Biol $2019 ; 29$ : R50-70.

10. Zimmerman $\varepsilon$, Maron J. Foxp2 gene deletion and infant feeding difficulties. Cold Spring Harb Mol Case Stud 2015 ; 20/6 Jan 2 : a000547.

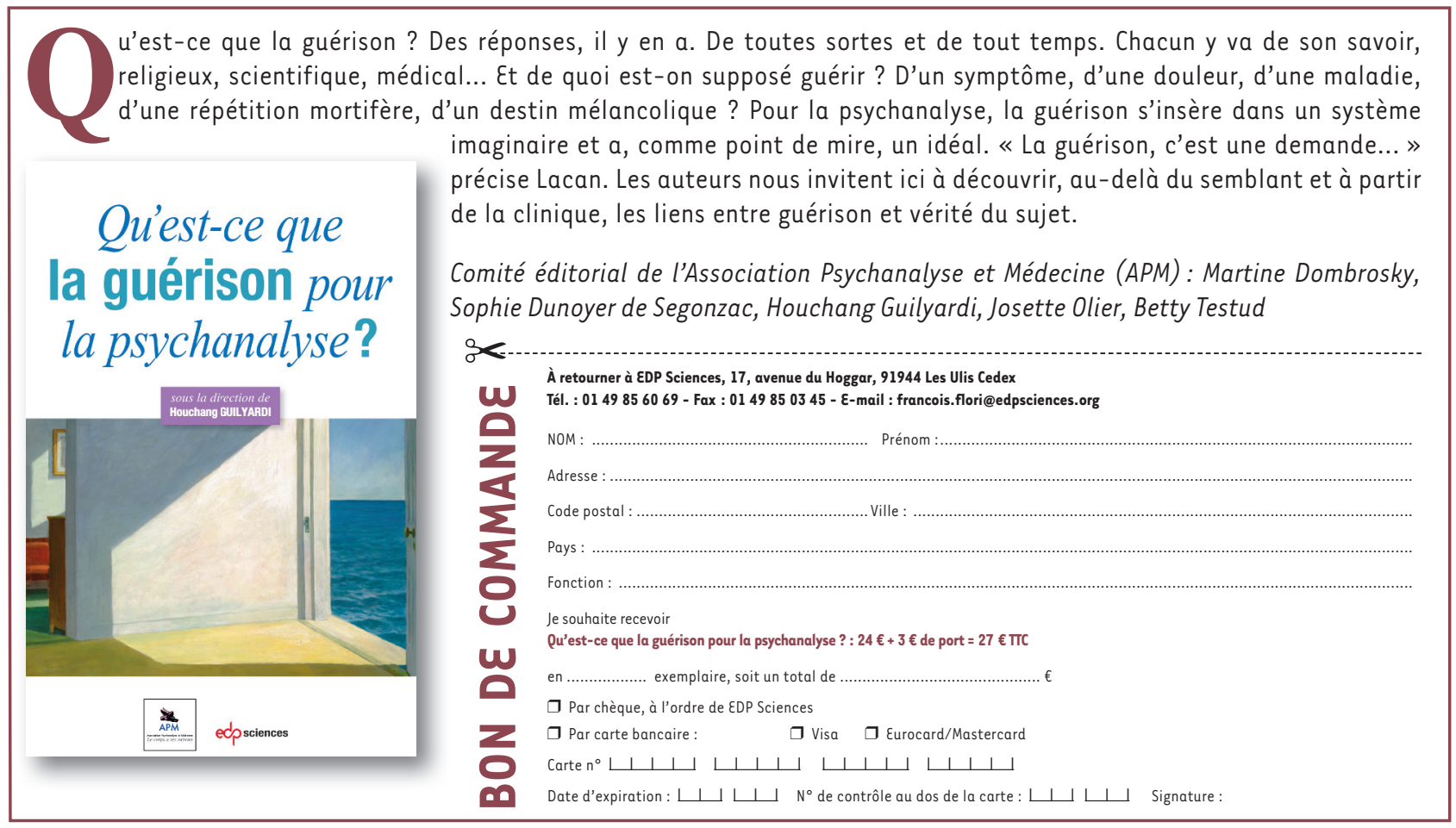

TIRÉS À PART

G. Couly

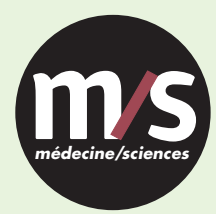

Tarifs d'abonnement $\mathrm{m} / \mathrm{s}-2020$

Abonnez-vous

à médecine/sciences
$>$ Grâce à $m / s$, vivez en direct les progrès des sciences biologiques et médicales

Bulletin d'abonnement page 186 dans ce numéro de $\mathrm{m} / \mathrm{s}$

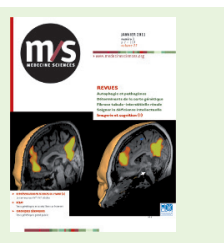

